\title{
Histología del anillo inguinal interno en pacientes con hernia inguinal indirecta: serie de casos observacional y descriptiva*
}

\author{
Drs. CARLOS BARRÍA M. ${ }^{3}$, PATRICIO AYALA S. ${ }^{2}$, VÍCTOR J. CORTÉS G. ${ }^{1}$, \\ LUIS JARAMILLO R. ${ }^{1,2}$, MARIO A. CONTRERAS G. ${ }^{1}$, CRISTÓBAL LARRAÍN T. ${ }^{1,2}$, \\ RODRIGO TAPIA P. ${ }^{1}$, E.U. KARINA S. CRUCES B. ${ }^{1}$ \\ Servicio de Cirugía, Hospital de La Serena. \\ Escuela de Medicina, Universidad Católica del Norte. \\ 3 Histomed. \\ La Serena, Chile.
}

\begin{abstract}
\section{Age associated changes in the histology of indirect inguinal hernia rings}

Background: This study has the purpose to investigate the microscopic basic histological structure of the internal inguinal ring in patients operated on for primary indirect inguinal hernia. Patients and Methods: A sample of 72 consecutive male patients older than 15 years-of-age with unilateral inguinal hernia submitted to elective surgery was studied. The primary outcome measure was the histological findings of the internal inguinal ring. All samples were processed only by histochemical techniques. Patients were divided in 3 groups according to age. Group I: patients between 15 and 40 years-of-age. Group II: patients between 41 and 70 years-of-age. Group III: patients older than 71 years. Results: All samples from the internal inguinal ring were constituted by fibrous fascial tissue with elastic fibers which were thicker in younger patients and thinner in older patients. Adipose tissue between elastic fibers was absent in younger patients and was abundant in older patients. Vascular sclerosis was minimal in Group I, moderate in Group II, and important in Group III. Acute or chronic inflammatory cells were absent in all patients. Conclusions: The histological characteristics of the internal inguinal ring in patients with indirect inguinal hernia consist on reduced density and thickness of elastic fibers and increased adipose tissue between elastic fibers. Vascular sclerosis was more severe as the age of the patients increased. These histological changes were related to normal aging.
\end{abstract}

Key words: Inguinal hernia, indirect hernia, indirect inguinal ring, hernia histology.

\section{Resumen}

Introducción: El presente estudio tiene como objetivo investigar la estructura histológica básica del anillo inguinal interno en pacientes operados por hernia inguinal indirecta primaria. Pacientes y Métodos: Se estudió una muestra de 72 pacientes consecutivos mayores de 15 años de edad con hernia inguinal indirecta

*Recibido el 1 de marzo de 2011 y aceptado para publicación el 6 de junio de 2011.

Clinical trials.gov - protocol registration system: NCT01211067

Los autores no reconocen conflictos de intereses - estudio financiado con fondos propios.

Correspondencia: Dr. Marcelo A. Beltrán S.

Manuel Antonio Caro 2629, La Serena, Chile. Casilla 912.

beltran_01@yahoo.com 
unilateral sometidos a cirugía electiva. El objetivo principal fue la histología básica del anillo inguinal interno. Las muestras fueron procesadas con técnicas histoquímicas. Los pacientes se dividieron en 3 grupos de acuerdo a la edad. Grupo I: pacientes entre 15 y 40 años de edad. Grupo II: pacientes entre 41 y 70 años. Grupo III: pacientes mayores de 71 años de edad. Resultados: Todas las muestras histológicas del anillo inguinal estuvieron constituidas por tejido fascial fibroso con fibras elásticas gruesas en pacientes jóvenes y delgadas en pacientes mayores. La esclerosis vascular fue mínima en el grupo I, moderada en el grupo II e importante en el grupo III. No se encontraron células inflamatorias agudas o crónicas. Conclusiones: Las características histológicas básicas del anillo inguinal interno consisten en fibras elásticas que disminuyen su densidad y se adelgazan con la edad y en un aumento simultáneo del tejido adiposo entre estas fibras. La esclerosis vascular se presenta y es más severa en la medida que la edad de los pacientes aumenta. Estos cambios histológicos se relacionan al envejecimiento normal.

Palabras clave: Hernia inguinal, hernia indirecta, anillo inguinal interno, histología herniaria.

\section{Introducción}

La histología básica del anillo inguinal interno no ha sido objeto tradicional de estudio entre los cirujanos dedicados a la cirugía herniaria. Desde el estudio experimental de Zolton T. Wirtschafter y J. Peter Bentley, postulando a los defectos en el metabolismo del colágeno como la causa de la formación de las hernias ${ }^{1}$; la investigación histológica, mediante el uso de técnicas de inmunohistoquímica y microscopia electrónica sobre la región inguinal en pacientes con hernia, se ha centrado en las alteraciones moleculares del metabolismo del colágeno que llevan a defectos de la ultraestructura en los tejidos tendinosos de la región inguinal y pared abdominal $^{2-15}$. Consecuentemente, la evidencia actual postula la noción de que la formación de las hernias primarias deriva de las deficiencias biológicas de la matriz extracelular, constituyendo una enfermedad del tejido conectivo ${ }^{15}$. Estudios investigando la histología básica del anillo inguinal interno en pacientes con hernia inguinal indirecta no han sido publicados sino recientemente ${ }^{16}$. Aún más, sólo existen algunos estudios antiguos y otros recientes que mencionan superficialmente algunas características histológicas básicas del anillo inguinal interno ${ }^{17-22}$. Se ha descrito la degeneración fibrohialina de las fibras musculares asociada a inflamación crónica y aguda en muestras histológicas del anillo inguinal interno de pacientes con hernia inguinal, sugiriendo que estos hallazgos constituyen la etiología de la hernia inguinal indirecta ${ }^{16}$. No estamos de acuerdo con esta hipótesis ${ }^{23}$. Sugerimos que las características histológicas básicas del anillo inguinal interno en pacientes con hernia inguinal indirecta y los cambios degenerativos que puedan observarse se deben al envejecimiento normal de los tejidos. Por lo tanto, estos cambios no constituyen parte de la etiología de la hernia. El objetivo del presente estudio es describir la estructura histológica básica del anillo inguinal interno en pacientes operados por hernia inguinal indirecta primaria.

\section{Pacientes y Métodos}

\section{Diseño del estudio y criterios de inclusión y exclusión}

El presente es un estudio prospectivo, observacional, descriptivo de casos consecutivos. Se incluyó una muestra de conveniencia constituida por 72 pacientes varones consecutivos mayores de 15 años de edad operados electivamente entre los meses de agosto de 2009 y febrero de 2010 en el Servicio de Cirugía del Hospital de La Serena. Los criterios de inclusión fueron: género masculino mayor de 15 años de edad; hernia inguinal indirecta primaria unilateral tipos I, II y III de Gilbert ${ }^{24}$; no-fumador; no-diabético; y ausencia de enfermedades del tejido conectivo tales como osteogénesis imperfecta, síndrome de Marfan, síndrome de Ehlers-Danlos, dislocación congénita de cadera de la infancia, riñón poliquístico autosómico dominante, e hipermobilidad articular. Los criterios de exclusión fueron: Género femenino; pacientes menores de 15 años de edad; hernia inguinal bilateral; hernia recurrente; hernia directa o mixta tipos IV y V de Gilbert ${ }^{24}$, o VI y VII de Rutkow y Robbins ${ }^{25}$; pacientes fumadores, diabéticos y portadores de las enfermedades del tejido conectivo previamente especificadas.

\section{Variable de resultado}

Las variables de resultado fueron los hallazgos histológicos del anillo inguinal interno.

\section{Variables y definiciones}

El tiempo de evolución de la hernia, se contó desde el inicio de los síntomas hasta el día de la cirugía. La obesidad se clasificó de acuerdo al Índice de Masa Corporal (IMC): Peso normal IMC menor a $29 \mathrm{~kg} / \mathrm{m}^{2}$, sobrepeso IMC entre 30 y $34 \mathrm{~kg} / \mathrm{m}^{2}$, obesidad IMC entre 35 y $40 \mathrm{~kg} / \mathrm{m}^{2}$, hiperobesidad IMC mayor a $41 \mathrm{~kg} / \mathrm{m}^{2}$. El tipo de trabajo de los pacientes se dividió en trabajo liviano cuando se trataba de trabajos de oficina o con mínimo esfuerzo físico y trabajo pesado cuando los pacientes eran 
agricultores o mineros. Se consideró como jubilado al paciente que no trabaja y se dedica sólo a las actividades de la vida diaria. Las estructuras histológicas fueron: fibras elásticas que se dividieron en delgadas y gruesas; tejido adiposo que se definió como presente o ausente; esclerosis vascular que se dividió en mínima, moderada y severa, hiperemia vascular definida como estasis de eritrocitos y otras células sanguíneas en el lumen de capilares arteriales o venosos; las células inflamatorias agudas fueron los polimorfonucleares y las células inflamatorias crónicas fueron los linfocitos y monocitos.

\section{Protocolo de toma de muestra y procesamiento}

Todos los pacientes firmaron un consentimiento informado en el cual accedían a participar en el estudio y a la toma de una muestra de tejido del anillo inguinal interno para biopsia. Todos los pacientes se operaron mediante la técnica de Lichtenstein ${ }^{26,27}$. Una vez que se confirmó la hernia como indirecta, el anillo inguinal interno fue apropiadamente disecado y entre dos ligaduras separadas entre sí por 1,5 a 2 $\mathrm{cm}$, se tomó una muestra de tejido (con tijeras o bisturí), de aproximadamente $1 \mathrm{~cm}$ x $0,5 \mathrm{~cm}$ del borde superior del pilar anterior del anillo. Las muestras se fijaron con formalina neutra al $10 \%$ y se enviaron para estudio histológico. La muestra se incluyó en un bloque de parafina y se cortó con el micrótomo en un grosor de 0,4 micrones. Para el análisis de las muestras se utilizaron únicamente técnicas histoquímicas: eosina y hematoxilina, van Gieson y orceina.

\section{Análisis de los grupos}

La serie se dividió en 3 grupos de acuerdo a la edad. Grupo I, constituido por 20 pacientes $(28 \%)$ entre 15 y 40 años de edad, el menor de los cuales tenía 16 años. Grupo II, constituido por 20 pacientes (28\%) entre 41 y 70 años. Grupo III, constituido por 32 pacientes (44\%) mayores de 71 años de edad, el mayor de los cuales tenía 85 años. La edad de los 2 primeros grupos fue definida arbitrariamente, la edad del grupo III, se definió de acuerdo a estudios previos en los que se utilizó este límite ${ }^{28}$.

\section{Estadística}

Las variables categóricas se expresaron con medidas de tendencia central y dispersión. Para las comparaciones entre grupos se utilizaron el $\chi^{2}$ y el T-test. Para comparar múltiples variables categóricas se utilizó el test no-paramétrico de Kruskall-Wallis. Las variables continuas entre grupos se compararon con el test de análisis de varianza de una vía. Se utilizó la regresión lineal para identificar variables que pudieran influenciar en los hallazgos histológicos.
Se utilizó el coeficiente de correlación parcial para encontrar correlaciones entre variables de acuerdo a los hallazgos histológicos. Se utilizó el programa SSPS versión 11.0 para el análisis estadístico.

\section{Resultados}

Los tres grupos de pacientes fueron comparables entre sí en términos estadísticos respecto al número de pacientes, promedio del IMC y patología asociada. Los pacientes de mayor edad tuvieron un mayor tiempo de evolución de la hernia. Todos los pacientes del grupo I y la mayoría en los grupos II y III, tuvieron un IMC entre 30 y $40 \mathrm{~kg} / \mathrm{m}^{2}$. Una proporción mayor de pacientes del grupo I tenían un trabajo liviano comparados con los grupos II y III. La mayoría de los pacientes de los grupos I y II tenían trabajo pesado comparado con el grupo III. Una mayor proporción de pacientes del grupo III estaban jubilados comparados con los grupos II y III. La mayoría de los pacientes de los grupos I y II no consumían alcohol, por el contrario, la mayoría de los pacientes del grupo III eran consumidores de alcohol. La hipertensión arterial fue más frecuente en los pacientes del grupo III. Otras patologías asociadas se presentaron con menor frecuencia en los grupos I y II (Tabla 1).

La histología del anillo inguinal interno se detalla en la Tabla 2. Todas las muestras estuvieron constituidas por tejido fibroso fascial con fibras elásticas gruesas en los pacientes jóvenes y delgadas en los pacientes ancianos. No se encontró tejido adiposo entre las fibras elásticas en los pacientes jóvenes; en los pacientes ancianos el tejido adiposo se encontró abundantemente entre estas fibras (Figuras 1, 2 y 3 ). La esclerosis vascular se encontró en los tres grupos; fue mínima en el grupo I, moderada en el grupo II e importante en el grupo III $(p<0,0001)$. Se encontró hiperemia en todos los pacientes. Todos los pacientes tenían paredes vasculares gruesas e hipercelulares, las cuales fueron más celulares en pacientes más jóvenes y más fibrosas en pacientes mayores (Figura 4). No se encontraron células inflamatorias agudas o crónicas en ninguna muestra. El análisis de regresión lineal identificó a la edad ( $\mathrm{p}<0,0001)$ como el único factor que influenció los hallazgos histológicos (Tabla 3). Los coeficientes de correlación parcial de acuerdo con la histología identificaron correlaciones positivas entre las siguientes variables: La edad se asocia al tiempo de evolución de la hernia y al tipo de trabajo de los pacientes. El IMC tiene una correlación positiva con las patologías asociadas y con la obesidad (Tabla 4). 


\section{Discusión}

Las características generales de los pacientes incluidos en el presente estudio, fueron las esperadas y no difieren de las series publicadas, el coeficiente de correlación parcial identificó correlaciones positivas entre variables en las que era esperable una correlación positiva en series de pacientes operados por hernia inguinal ${ }^{26-32}$. Estos resultados validan la presente serie de pacientes como una muestra adecuada para estudiar la histología del anillo inguinal interno.

El piso del canal inguinal está compuesto de dos láminas fasciales íntimamente adherentes. La fascia superficial, gruesa y resistente, está formada por extensiones aponeuróticas de la fascia del músculo transverso; inmediatamente por debajo se encuentra la fascia transversalis, delgada y fibrosa $a^{21,33-37}$. El anillo inguinal interno constituye una abertura en el piso del canal inguinal ${ }^{17-21,36,37}$. Esta abertura es gruesa en sus bordes y está rodeada por fibras fasciales del músculo transverso y del tendón conjunto y por fibras musculares del músculo oblicuo interno ${ }^{17,20,21,34,36,37}$. El anillo inguinal interno es un anillo incompleto compuesto por dos pilares, un pilar anterior largo y un pilar posterior corto, el pilar anterior se fija al músculo transverso abdominal y su aponeurosis y el pilar posterior se inserta en el tracto iliopúbico, formando una estructura en " $U$ " inverti$\mathrm{da}^{20,36,37}$. Cuando el músculo transverso se contrae, el anillo se cierra por detrás del borde muscular del músculo oblicuo interno ${ }^{20,34,35,37}$. Las fibras aponeuróticas inferiores del músculo oblicuo interno se originan en el ligamento inguinal y se curvan hacia arriba, adentro y abajo para formar el tendón conjunto ${ }^{20,37}$. La contracción de estas fibras aproxima el borde muscular del tendón conjunto y el ligamento inguinal, reforzando el anillo inguinal interno contra aumentos de la presión intra-abdominal ${ }^{17,20,34,35,37}$. Las muestras de tejido se tomaron del pilar anterior del anillo inguinal. Las biopsias mostraron una arquitectura histológica normal con cambios degenerativos asociados al envejecimiento de los pacientes, que se hace evidente por la marcada disminución de fibras elásticas en grosor y densidad; la aparición e incremento de tejido adiposo entre las fibras elásticas; y el aumento de la esclerosis vascular en pacientes de mayor edad, siendo el resultado una menor elasticidad de la fascia. Estos cambios degenerativos son los esperados con la edad avanzada en tejidos fasciales constituidos por fibras elásticas y fibras de colágeno, como se sugirió inespecíficamente en otros reportes ${ }^{5,22,35}$.

Otro hallazgo interesante fue el engrosamiento de las paredes vasculares en pacientes de todas las edades, sugiriendo que los tejidos de la región inguinal se encuentran constantemente sometidos a elevadas presiones. Esta característica histológica de la región inguinal en pacientes con hernia inguinal probablemente se debe a las altas presiones intra-abdominales en estos pacientes, la presión intra-abdominal elevada es un factor etiológico en la formación de la hernia ${ }^{34,35,37}$. Respecto a las células inflamatorias agudas o crónicas previamente reportadas ${ }^{16}$, en el presente estudio no se encontraron en ninguna de las muestras histológicamente estudiadas, consecuentemente nuestros hallazgos no apoyan tal noción.

La edad avanzada, es otro factor que predispone al desarrollo de las hernias mediante un mecanismo que incluye la reducción de las fibras oxitalánicas que son responsables de la resistencia tisular y por el incremento en las fibras maduras de elastina y elaunina, las que son responsables por la elasticidad tisular ${ }^{5}$. Estos cambios secundarios a la edad avanzada junto con los cambios estructurales en el grosor, acortamiento y enrollamiento de las fibras elásticas maduras, inducen la pérdida de la función elástica de estas fibras, lo que ha llevado a argüir que el envejecimiento del anillo inguinal interno y del músculo oblicuo interno predispone al desarrollo de la hernia inguinal ${ }^{5}$. Sin embargo, otros autores arguyen que todas las hernias inguinales indirectas en adultos e infantes son congénitas ${ }^{38}$. Ambos argumentos son aún motivo de acalorada controversia. Los resultados del presente estudio contribuyen a esta controversia al demostrar que el anillo inguinal interno sufre cambios histológicos degenerativos con la edad avanzada y sin embargo, la hernia inguinal indirecta se presenta en todas las edades.

Los factores de riesgo conocidos que predisponen al desarrollo de hernias inguinales y de otros tipos, se relacionan al número y grosor de las fibras de colágeno en los tejidos fasciales ${ }^{39}$. En pacientes con hernia inguinal se observan consistentemente tejidos fasciales muy atenuados o incluso la ausencia de los mismos ${ }^{19,34,39,40}$. Los defectos del metabolismo y calidad de las fibras de colágeno tipos I y III predisponen al desarrollo de hernia inguinal ${ }^{2-7,15,40}$. La hidroxiprolina, que es un producto del colágeno y que contiene prolina y lisina, es esencial para el entrecruzamiento intermolecular e intramolecular y para la glicosilación del colágeno; y se encuentra disminuida en pacientes con hernia inguina ${ }^{40}$. Otros factores conocidos que influyen en el desarrollo de hernias inguinales son las enfermedades del tejido conectivo, la activación de las colagenasas y la activación e incremento de los niveles de elastasa y metaloproteinasa en fumadores ${ }^{40}$. Con la intención de seleccionar una muestra sin factores conocidos para el desarrollo de hernia inguinal, en el presente estudio se excluyeron a los fumadores y a los pacientes con patología conocida del tejido conectivo. Consecuentemente, los hallazgos histológicos de- 
mostrando cambios degenerativos del anillo inguinal interno relacionados con la edad, establecen estas características histológicas como las que se deben esperar en esta condición.

\section{Conclusiones}

Las características histológicas básicas del anillo inguinal interno en pacientes con hernia inguinal interna consisten en densidad y grosor disminuido de las fibras elásticas, aumento del tejido adiposo entre estas fibras, desarrollo de esclerosis vascular y engrosamiento muscular de las paredes vasculares que se manifiestan con el envejecimiento normal de los pacientes.

\section{Referencias}

1. Wirtschafter ZT, Bentley JP. Hernias as a collagen maturation defect. Ann Surg.1964:160:852-9.

2. Klinge U, Binnebösel M, Mertens PR. Are collagens the culprits in the development of incisional and inguinal hernia disease? Hernia 2006;10:472-7.

3. Pans A, Albert A, Lapière CM, Nusgens B. Biochemical study of collagen in adult groin hernias. J Surg Res. 2001;95:107-13.

4. Friedman DW, Boyd CD, Norton P, Greco RS, Boyarsky AH, Mackenzie JW, et al. Increases in type III collagen gene expression and protein synthesis in patients with inguinal hernias. Ann Surg. 1993;218:754-60.

5. Quintas ML, Rodrigues CJ, Yoo JH, Rodrigues Junior AJ. Age related changes in the elastic fiber system of the interfoveolar ligament. Rev Hosp Clin Fac Med Sao Paulo 2000;55:83-6.

6. Rodrigues Junior AJ, Rodrigues CJ, da Cunha AC, Yoo JH. Quantitative analysis of collagen and elastic fibers in the transversalis fascia in direct and indirect inguinal hernia. Rev Hosp Clin Fac Med Sao Paulo 2002;57:26570 .

7. Goldstein SL. Mechanism and metabolic characteristics of hernia formation. Prob Gen Surg. 2002;19:1-6.

8. Bórquez PM, Garrido LO, Manterola CD, Peña PS, Schlageter CT, Orellana JJ, et al. Collagen and elastic fibers of skin connective tissue in patients with and without primary inguinal hernia. Rev Med Chile 2003;131:1273-9.

9. Bórquez PM. Estudio inmunohistoquímico del colágeno tipo I y tipo III en piel de pacientes con hernia inguinal primaria. Rev Chil Cir. 2005;57:489-94.

10. Bórquez PM, Garrido LO, Peña PS. Fisiopatología de la hernia inguinal primaria, algo más que un fenómeno mecánico. Rev Chil Cir. 2005;57:432-5.

11. Szczęsny W, Cerkaska K, Tretyn A, Dąbrowiecki S. Etiology of inguinal hernia: Ultrastructure of rectus sheath revisited. Hernia 2006;10:266-71.
12. Franz MG. The biology of hernias and abdominal wall. Hernia 2006;10:462-71.

13. Meyer AL, Berger E, Monteiro Jr. O, Alonso PA, Stavale JN, Gonçalves MP. Quantitative and qualitative analysis of collagen types in the fascia transversalis of inguinal hernia patients. Arq Gastroenterol. 2007;44:230-4.

14. Casanova AB, Trindade EN, Trindade MR. Collagen in the transversalis fascia of patients with indirect inguinal hernia: A case-control study. Am J Surg. 2009;198:1-5.

15. Franz MG. The biology of hernia formation. Surg Clin North Am. 2008;88:1-15.

16. Amato G, Marasa L, Sciacchitano T, Bell SG, Romano $\mathrm{G}$, Gioviale MC, et al. Histological findings of the internal inguinal ring in patients having indirect inguinal hernia. Hernia 2009; 13:259-62.

17. Dickson AR. The inguinal ring in oblique inguinal hernia. Cal West Med. 1931;35:204-7.

18. Lunn HF. The anatomy of inguinal hernia. Ann R Coll Surg Engl. 1948;2:285-98.

19. Peacock EE, Madden JW. Studies on the biology and treatment of recurrent inguinal hernia: II. Morphological changes. Ann Surg. 1974;179:567-71.

20. Skandalakis JE, Gray SW, Skandalakis LJ, Colborn GL, Pemberton LB. Surgical anatomy of the inguinal area. World J Surg. 1998;13:490-8.

21. Menck J, Lierse W. The fascia of the inguinal canal ring. Chirurg. 1991; 62:117-20.

22. Feleshtyns'kyı̆ IaP, Hrabovyı̆ OM, Chyn'ba OV. Differentiated approach to surgical treatment of inguinal hernia in accordance with morphologic characteristics of musculo-aponeurotic structures of inguinal channel. Klin Khir. 2007;10:22-6.

23. Barría C, Beltrán MA, Contreras MA, Cruces KS. Histology of the internal inguinal ring: It is really a novelty? Hernia 2010;14:339-40.

24. Gilbert AI. An anatomic and functional classification for the diagnosis and treatment of inguinal hernia. Am J Surg. 1989;157:331-34.

25. Rutkow IM, Robbins AW. "Tension-free" inguinal herniorrhaphy: A preliminary report on the "Mesh-Plug" technique. Surgery 1993;114:3-7.

26. Lichtenstein IL, Shulman AG, Amid PK, Montflor MM. The tension-free hernioplasty. Am J Surg. 1989;157:188-93.

27. Wantz GE. Experience with the tension-free hernioplasty for primary inguinal hernia in men. J Am Coll Surg. 1996;183:351-6.

28. Beltrán MA, Cruces KS. The outcomes of open tension-free hernioplasty in elderly patients. Hernia 2006;10:395-400.

29. Beltrán MA, Burgos C, Almonacid J, Tapia TF, Vicencio A, Danilova T, et al. Long-term follow-up of tension-free Lichtenstein hernioplasty: Application of a qualitative-and-quantitative measurement instrument. Hernia 2005;9:368-74. 
30. Beltrán MA, Cruces KS. Outcomes of Lichtenstein hernioplasty for primary and recurrent inguinal hernia. World J Surg. 2006;30:2281-7.

31. Hernández-Granados P, Quintáns-Rodríguez A. Early complications in tension-free hernioplasty: Comparison between ambulatory and short-stay surgery. Hernia 2000;4:238-41.

32. Hernández-Granados $\mathrm{P}$, Ontañon $\mathrm{M}$, Lasala $\mathrm{M}$, García C, Arguello M, Medina I. Tension-free hernioplasty in primary inguinal hernias: A series of 2054 cases. Hernia 2000;4:141-3.

33. Madden JL, Hakim S, Agorogiannis AB. The anatomy and repair of inguinal hernias. Surg Clin N Am. 1971;51:1269-92.

34. Desarda MP. Surgical physiology of inguinal hernia repair - A study of 200 cases. BMC Surgery 2003;3:2.

35. Desarda MP. Physiological repair of inguinal her- nia: A new technique (Study of 860 repairs). Hernia 2006;10:143-6.

36. Arregui ME. Surgical anatomy of the preperitoneal fasciae and posterior transversalis fasciae in the inguinal region. Hernia 1997;1:101-10.

37. Peiper C, Junge K, Prescher A, Stumpf M, Schumpelick $\mathrm{V}$. Abdominal musculature and the transversalis fascia: An anatomical viewpoint. Hernia 2004;8:376-80.

38. van Wessem KJ, Simons MP, Plaisier PW, Lange JF. The etiology of indirect inguinal hernias: Congenital and / or acquired? Hernia 2003; 7:76-9.

39. Wolloscheck T, Gaumann A, Terzic A, Heintz A, Junginger Th. Konerding MA. Inguinal hernia: Measurement of the biomechanics of the lower abdominal wall and the inguinal canal. Hernia 2004;8:233-41.

40. Bendavid R. The unified theory of hernia formation. Hernia 2004;8:171-6. 